\title{
A new fourth-order integrable nonlinear equation: breather, rogue waves, other lump interaction phenomena, and conservation laws
}

\author{
Dumitru Baleanu ${ }^{1,2}$, Ali Saleh Alshomrani ${ }^{3}$ and Malik Zaka Ullah ${ }^{3 *}$
}

\author{
"Correspondence: \\ malikzakas@gmail.com \\ ${ }^{3}$ Mathematical Modelling and \\ Applied Computation Research \\ Group (MMAC), Department of \\ Mathematics, Faculty of Science, \\ King Abdulaziz University, Jeddah \\ 21589, Saudi Arabia \\ Full list of author information is \\ available at the end of the article
}

\begin{abstract}
In this study, we investigate a new fourth-order integrable nonlinear equation. Firstly, by means of the efficient Hirota bilinear approach, we establish novel types of solutions which include breather, rogue, and three-wave solutions. Secondly, with the aid of Lie symmetry method, we report the invariance properties of the studied equation such as the group of transformations, commutator and adjoint representation tables. A differential substitution is found by nonlinear self-adjointness (NSA) and thereafter the associated conservation laws are established. We show some dynamical characteristics of the obtained solutions through via the 3-dimensional and contour graphs.
\end{abstract}

Keywords: Fourth-order integrable nonlinear equation; Lump solutions; Interaction solutions; Invariant analysis; Conservation laws

\section{Introduction}

In differential equation (DE) concepts, Cauchy problem (CP) is considered as one of the most fundamental problems to analyze a solution of a DE which satisfies initial data. Classical methods, like the Laplace and Fourier transformation methods, have been introduce to solve CPs for linear partial and ordinary DEs. The isomonodromic and inverse scattering approaches were created to handle CPs for nonlinear partial and ordinary DEs, respectively [1-3]. A captivating and excellent field of study is the analysis of the exact solutions (ESs) and the problems of constructing solutions for an expansive range of nonlinear equations.

The ESs for partial DEs describe important physical and mathematical aspects. A soliton solution is an ES that is investigated by exponentially located functions that move in all directions in both time and space. Also, a lump solution can be regarded as an exact solution of a partial DE, obtained by taking long wave limits from soliton theory [3]. Nonetheless, only in space, a lump solution can be localized in all directions. Additionally, it is well known that more nonlinear phenomena can be described by interaction solutions

(c) The Author(s) 2021. This article is licensed under a Creative Commons Attribution 4.0 International License, which permits use sharing, adaptation, distribution and reproduction in any medium or format, as long as you give appropriate credit to the original author(s) and the source, provide a link to the Creative Commons licence, and indicate if changes were made. The images or other third party material in this article are included in the article's Creative Commons licence, unless indicated otherwise in a credit line to the material. If material is not included in the article's Creative Commons licence and your intended use is not permitted by statutory regulation or exceeds the permitted use, you will need to obtain permission directly from the copyright holder. To view a copy of this licence, visit http://creativecommons.org/licenses/by/4.0/ 
between lump and soliton solutions. Nevertheless, the properties of the interaction are seldom debated, as the mathematical calculation involved is much more complex.

Over the last two decades, several researchers have studied solitary solutions, lump solutions, and other types of integrable equation solutions. This includes the Ishimori-I equation [4], the Davey-Stewarton equation II [3], the BKP equation [5, 6], three-dimensional three-wave resonant interaction [7], and self-consistent KP equation [8]. Many nonintegrable equations do have lump solutions, such as the generalized KP and Sawada-Kotera equations [9-12]. Through important properties of lump solutions, it can be understood that amplitudes, shapes, speeds of solitons will be preserved after collision with another soliton, and this is the elastic property of a collision. Moreover, interactions between rouge, breather, three-wave, and kink solitary wave solutions have been established in [13-15]. In addition, various researches demonstrate the existence of interaction solutions between lumps and other types of specific solutions to a nonlinear integrable equation [16-19]. Furthermore, in order to justify the existence and uniqueness, some important internal properties, as well as the integrability of a DE, computing conservation laws and symmetries are some of the best aspects many scientists employ to do the job [20-26]. Therefore, establishing lumps solutions, their interactions, as well as the conservation laws for various types of DE, are of humongous importance.

This study is aimed at using the Hirota bilinear approach [14] to construct some novel breather, rogue, and three-wave solutions to a new integrable fourth-order nonlinear equation. On the other hand, the Lie symmetry analysis [27] is going to be used to generate the conservation laws for this nonlinear equation.

The new integrable fourth-order nonlinear equation is given by [28]

$$
\Xi_{t t}+\Xi_{t x x x}+\alpha\left(\Xi_{x} \Xi_{t}\right)_{x}=0
$$

where $\alpha$ is the coefficient of the nonlinear term $\left(\Xi_{x} \Xi_{t}\right)_{x}$. Nonlinearity arises when the change of the output is not proportional to the change of the input [29].

\section{Lump interaction phenomena}

In this section, we construct some novel breather, rogue, and three-wave solutions to Eq. (1).

Applying the Cole-Hopf transformation [28, 30]

$$
\Xi(x, t)=\frac{6}{\alpha}(\ln f(x, t))_{x}
$$

to Eq. (1) yields the following bilinear form:

$$
3 f_{x t} f_{x x}-3 f_{x} f_{x x x t}-f_{t}\left(f_{t}+f_{x x x}\right)+f\left(f_{t t}+f_{x x x t}\right)=0 .
$$

\subsection{Breather waves}

In this subsection, we construct the breather wave solutions to Eq. (1).

Consider the following test function [31-33] as a solution to the bilinear equation (3):

$$
f(x, t)=e^{\xi_{1}}+m_{1} \cos \left(\xi_{2}\right)+m_{2} e^{\xi_{3}},
$$

where $\xi_{1}=-p_{1}\left(a_{0} t+x\right), \xi_{2}=p_{0}\left(b_{0} t+x\right)$, and $\xi_{3}=p_{1}\left(a_{0} t+x\right)$. 
Substituting Eq. (4) into Eq. (3) gives a polynomial in the powers of trigonometric and exponential functions. Collecting the coefficients of the same power and equating each sum to zero yields an algebraic system of equations. We solve this system of equations to obtain the values of the parameters involved. Substituting the values of the parameters into Eq. (2) gives the following breather wave solutions to Eq. (1):

Case-1: When

$$
p_{1}=\frac{\sqrt{-8 a_{0} b_{0}+3 a_{0}^{2}-3 b_{0}^{2}}}{\sqrt{24 a_{0}+8 b_{0}}}, \quad p_{0}=-\frac{\sqrt{3 a_{0}-b_{0}}}{2 \sqrt{2}}, \quad m_{2}=-\frac{b_{0} m_{1}^{2}\left(3 a_{0}-b_{0}\right)}{4 a_{0}\left(a_{0}-3 b_{0}\right)}
$$

we get

$$
\begin{aligned}
f_{1}(x, t)= & -\frac{b_{0} m_{1}^{2}\left(3 a_{0}-b_{0}\right) \mathrm{e}^{\left(\frac{\sqrt{-8 a_{0} b_{0}+3 a_{0}^{2}-3 b_{0}^{2}}\left(a_{0} t+x\right)}{\sqrt{24 a_{0}+8 b_{0}}}\right)}}{4 a_{0}\left(a_{0}-3 b_{0}\right)} \\
& +\mathrm{e}^{\left(-\frac{\sqrt{-8 a_{0} b_{0}+3 a_{0}^{2}-3 b_{0}^{2}}\left(a_{0} t+x\right)}{\sqrt{24 a_{0}+8 b_{0}}}\right)}+m_{1} \cos \left(\frac{\sqrt{3 a_{0}-b_{0}}\left(b_{0} t+x\right)}{2 \sqrt{2}}\right) .
\end{aligned}
$$

Thus,

$$
\begin{aligned}
& \Xi_{1}(x, t) \\
& =\frac{6\left(-\frac{\mathrm{e}^{-\Theta} \sqrt{-8 a_{0} b_{0}+3 a_{0}^{2}-3 b_{0}^{2}}}{\sqrt{24 a_{0}+8 b_{0}}}-\frac{b_{0} \mathrm{e}^{\Theta} 1 m_{1}^{2}\left(3 a_{0}-b_{0}\right) \sqrt{-8 a_{0} b_{0}+3 a_{0}^{2}-3 b_{0}^{2}}}{4 a_{0}\left(a_{0}-3 b_{0}\right) \sqrt{24 a_{0}+8 b_{0}}}-\frac{m_{1} \sqrt{3 a_{0}-b_{0}} \sin \left(\Theta_{2}\right)}{2 \sqrt{2}}\right)}{\alpha\left(-\frac{b_{0} \mathrm{e}^{\Theta} m_{1}^{2}\left(3 a_{0}-b_{0}\right)}{4 a_{0}\left(a_{0}-3 b_{0}\right)}+\mathrm{e}^{-\Theta_{1}}+m_{1} \cos \left(\Theta_{2}\right)\right)},
\end{aligned}
$$

where

$$
\Theta_{1}=\frac{\sqrt{-8 a_{0} b_{0}+3 a_{0}^{2}-3 b_{0}^{2}}\left(a_{0} t+x\right)}{\sqrt{24 a_{0}+8 b_{0}}}
$$

and

$$
\Theta_{2}=\frac{\sqrt{3 a_{0}-b_{0}}\left(b_{0} t+x\right)}{2 \sqrt{2}}
$$

Case-2: When

$$
\begin{aligned}
a_{0}= & \frac{p_{1}^{6}-6 p_{0}^{2} p_{1}^{4}+9 p_{0}^{4} p_{1}^{2}-3 \sqrt{-p_{0}^{2} p_{1}^{2}\left(3 p_{0}^{2}-p_{1}^{2}\right)^{2}} p_{1}^{2}+\sqrt{-p_{0}^{2} p_{1}^{2}\left(3 p_{0}^{2}-p_{1}^{2}\right)^{2}} p_{0}^{2}}{2\left(3 p_{0}^{2} p_{1}^{2}-p_{1}^{4}\right)}, \\
b_{0}= & \frac{p_{0}^{4}-3 p_{1}^{2} p_{0}^{2}-\sqrt{-9 p_{1}^{2} p_{0}^{6}+6 p_{1}^{4} p_{0}^{4}-p_{1}^{6} p_{0}^{2}}}{2 p_{0}^{2}}, \\
m_{2}= & \left(-7 m_{1}^{2} p_{1}^{8}+55 m_{1}^{2} p_{0}^{2} p_{1}^{6}-109 m_{1}^{2} p_{0}^{4} p_{1}^{4}+24 m_{1}^{2} \sqrt{-p_{0}^{2} p_{1}^{2}\left(3 p_{0}^{2}-p_{1}^{2}\right)^{2}} p_{1}^{4}\right. \\
& \left.+21 m_{1}^{2} p_{0}^{6} p_{1}^{2}+24 m_{1}^{2} \sqrt{-p_{0}^{2} p_{1}^{2}\left(3 p_{0}^{2}-p_{1}^{2}\right)^{2}} p_{0}^{2} p_{1}^{2}\right) \\
& /\left(4\left(-49 p_{1}^{8}+145 p_{0}^{2} p_{1}^{6}+5 p_{0}^{4} p_{1}^{4}+3 p_{0}^{6} p_{1}^{2}\right)\right),
\end{aligned}
$$


we get

$$
\begin{aligned}
f_{2}(x, t)= & \mathrm{e}^{-\Theta_{3}}+m_{1} \cos \left(\Theta_{4}\right) \\
& +\frac{\mathrm{e}^{\Theta_{3}}\left(-\Theta_{5}+\Theta_{6}+21 m_{1}^{2} p_{1}^{2} p_{0}^{6}-109 m_{1}^{2} p_{1}^{4} p_{0}^{4}+55 m_{1}^{2} p_{1}^{6} p_{0}^{2}\right)}{4\left(-49 p_{1}^{8}+145 p_{0}^{2} p_{1}^{6}+5 p_{0}^{4} p_{1}^{4}+3 p_{0}^{6} p_{1}^{2}\right)} .
\end{aligned}
$$

Thus,

$$
\Xi_{2}(x, t)=\frac{6\left(m_{1} p_{0}\left(-\sin \left(\Theta_{4}\right)\right)-\mathrm{e}^{-\Theta_{3}} p_{1}+\frac{\mathrm{e}^{\Theta_{3}}\left(-\Theta_{5}+\Theta_{6}+\Theta_{7}\right) p_{1}}{\Theta_{8}}\right)}{\alpha\left(\mathrm{e}^{-\Theta_{3}}+\frac{\mathrm{e}^{\Theta}\left(-\Theta_{5}+\Theta_{6}+\Theta_{7}\right)}{\Theta_{8}}+m_{1} \cos \left(\Theta_{4}\right)\right)},
$$

where

$$
\begin{aligned}
& \Theta_{3}=p_{1}\left(\frac{\left(p_{1}^{6}-6 p_{0}^{2} p_{1}^{4}+9 p_{0}^{4} p_{1}^{2}-3 \sqrt{-p_{0}^{2} p_{1}^{2}\left(3 p_{0}^{2}-p_{1}^{2}\right)^{2}} p_{1}^{2}+\sqrt{-p_{0}^{2} p_{1}^{2}\left(3 p_{0}^{2}-p_{1}^{2}\right)^{2}} p_{0}^{2}\right) t}{2\left(3 p_{0}^{2} p_{1}^{2}-p_{1}^{4}\right)}+x\right), \\
& \Theta_{4}=p_{0}\left(\frac{\left(p_{0}^{4}-3 p_{1}^{2} p_{0}^{2}-\sqrt{\left.-9 p_{1}^{2} p_{0}^{6}+6 p_{1}^{4} p_{0}^{4}-p_{1}^{6} p_{0}^{2}\right) t}\right.}{2 p_{0}^{2}}+x\right), \\
& \Theta_{5}=7 m_{1}^{2} p_{1}^{8}-24 m_{1}^{2} p_{0}^{2} p_{1}^{2} \sqrt{-p_{0}^{2} p_{1}^{2}\left(3 p_{0}^{2}-p_{1}^{2}\right)^{2}} \\
& \Theta_{6}=24 m_{1}^{2} p_{1}^{4} \sqrt{-p_{0}^{2} p_{1}^{2}\left(3 p_{0}^{2}-p_{1}^{2}\right)^{2}} \\
& \Theta_{7}=21 m_{1}^{2} p_{1}^{2} p_{0}^{6}-109 m_{1}^{2} p_{1}^{4} p_{0}^{4}+55 m_{1}^{2} p_{1}^{6} p_{0}^{2}, \\
& \Theta_{8}=4\left(-49 p_{1}^{8}+145 p_{0}^{2} p_{1}^{6}+5 p_{0}^{4} p_{1}^{4}+3 p_{0}^{6} p_{1}^{2}\right) .
\end{aligned}
$$

\subsection{Rogue waves}

In this subsection, we construct the rogue wave solutions to Eq. (1). Consider the following test function [34] as a solution to the bilinear equation (3):

$$
f(x, t)=\xi_{1}^{2}+\xi_{2}^{2}+b_{7}+T \cosh \left(\xi_{3}\right)
$$

where $\xi_{1}=b_{1} t+b_{2} x+b_{3}, \xi_{2}=b_{4} t+b_{5} x+b_{6}$, and $\xi_{3}=T_{1} t+T_{2} x$.

Substituting Eq. (9) into Eq. (3) gives a polynomial in the powers of $x$, $t$, and hyperbolic functions. Collecting the coefficients of the same power and equating each sum to zero yields an algebraic system of equations. We solve this system of equations to obtain the values of the parameters involved. Putting the values of the parameters into Eq. (2) gives the following rogue waves solution to Eq. (1):

When

$$
b_{1}=-\frac{b_{2}}{3 T_{1}^{2}}, \quad b_{4}=-\frac{\mathrm{i} b_{2}}{3 T_{1}^{2}}, \quad b_{5}=\mathrm{i} b_{2}, \quad b_{6}=\frac{\sqrt{3} T T_{1}^{3}+2 \mathrm{i} b_{2} b_{3}}{2 b_{2}}, \quad T_{2}=-T_{1}^{3},
$$

we have

$$
f_{1}(x, t)=\left(b_{2} t-\frac{b_{2} x}{3 T_{1}^{2}}+b_{3}\right)^{2}+b_{7}+\Theta_{10}^{2}+T \cosh \left(T_{1} x-t T_{1}^{3}\right)
$$


Thus,

$$
\Xi_{1}(x, t)=\frac{6\left(-\frac{2 b_{2}\left(b_{2} t-\frac{b_{2} x}{3 T_{1}^{2}}+b_{3}\right)}{3 T_{1}^{2}}-\Theta_{9}+T T_{1} \sinh \left(T_{1} x-t T_{1}^{3}\right)\right)}{\alpha\left(\left(b_{2} t-\frac{b_{2} x}{3 T_{1}^{2}}+b_{3}\right)^{2}+b_{7}+\Theta_{10}^{2}+T \cosh \left(T_{1} x-t T_{1}^{3}\right)\right)},
$$

where

$$
\begin{aligned}
& \Theta_{9}=\frac{2 i b_{2}\left(i b_{2} t+\frac{\sqrt{3} T T_{1}^{3}+2 i b_{2} b_{3}}{2 b_{2}}-\frac{i b_{2} x}{3 T_{1}^{2}}\right)}{3 T_{1}^{2}}, \\
& \Theta_{10}=i b_{2} t+\frac{\sqrt{3} T T_{1}^{3}+2 i b_{2} b_{3}}{2 b_{2}}-\frac{i b_{2} x}{3 T_{1}^{2}} .
\end{aligned}
$$

\subsection{Three-wave solutions}

In this subsection, the three-wave solutions to Eq. (1) are revealed.

Consider the following test function [35] as a solution to the bilinear equation (3):

$$
f(\xi, z, t)=c_{1} \mathrm{e}^{\xi_{1}}+c_{2} \mathrm{e}^{-\xi_{1}}+c_{3} \sin \left(\xi_{2}\right)+c_{4} \sinh \left(\xi_{3}\right),
$$

where $\xi_{1}=b_{1} x+b_{2} t, \xi_{2}=b_{3} x+b_{4} t$, and $\xi_{3}=b_{5} x+b_{6} t$.

Substituting Eq. (12) into Eq. (3) gives a polynomial in the powers of trigonometric, hyperbolic, and exponential functions. Collecting the coefficients of the same power and equating each sum to zero provides an algebraic system of equations. We solve this system of equations to obtain the values of the parameters involved. Putting the values of the parameters into Eq. (2) produces the following wave solutions to Eq. (1):

Case-1: When

$$
\begin{aligned}
& b_{1}=\sqrt{4 \sqrt{3} b_{3}^{2}-7 b_{3}^{2}}, \quad b_{4}=3 \sqrt{3} b_{3}^{3}-5 b_{3}^{3}, \quad c_{3}=2 \sqrt{7 c_{1} c_{2}-4 \sqrt{3} c_{1} c_{2}}, \quad c_{4}=0 \\
& b_{2}=\frac{1}{4}\left(-\left((4 \sqrt{3}-7) b_{3}^{2}\right)^{3 / 2}-3 \sqrt{(4 \sqrt{3}-7) b_{3}^{2}} b_{3}^{2}\right)
\end{aligned}
$$

we have

$$
f_{1}(x, t)=c_{1} e^{\Theta_{11}}+c_{2} e^{-\Theta_{11}}+2 \sqrt{7 c_{1} c_{2}-4 \sqrt{3} c_{1} c_{2}} \sin \left(\Theta_{12}\right) .
$$

Thus,

$$
\begin{aligned}
\Xi_{1}(x, t)= & \left(6 \left(\sqrt{4 \sqrt{3} b_{3}^{2}-7 b_{3}^{2}} c_{1} \mathrm{e}^{\Theta 11}-\sqrt{4 \sqrt{3} b_{3}^{2}-7 b_{3}^{2}} c_{2} \mathrm{e}^{-\Theta_{11}}\right.\right. \\
& \left.\left.+2 b_{3} \sqrt{7 c_{1} c_{2}-4 \sqrt{3} c_{1} c_{2}} \cos \left(\Theta_{12}\right)\right)\right) \\
& /\left(\alpha\left(c_{1} \mathrm{e}^{\Theta_{11}}+c_{2} \mathrm{e}^{-\Theta_{11}}+2 \sqrt{7 c_{1} c_{2}-4 \sqrt{3} c_{1} c_{2}} \sin \left(\Theta_{12}\right)\right)\right),
\end{aligned}
$$

where

$$
\Theta_{11}=\frac{1}{4}\left(-\left((4 \sqrt{3}-7) b_{3}^{2}\right)^{3 / 2}-3 \sqrt{(4 \sqrt{3}-7) b_{3}^{2}} b_{3}^{2}\right) t+\sqrt{4 \sqrt{3} b_{3}^{2}-7 b_{3}^{2} x}
$$


and

$$
\Theta_{12}=\left(3 \sqrt{3} b_{3}^{3}-5 b_{3}^{3}\right) t+b_{3} x .
$$

Case-2: When

$$
b_{1}=-\sqrt[3]{2} \sqrt[3]{b_{6}}-b_{5}, \quad b_{2}=b_{6}, \quad c_{3}=0, \quad c_{4}=\frac{2 \sqrt{\Theta_{13}}}{\sqrt{16 b_{5}^{6} b_{6}-b_{6}^{3}}}
$$

we have

$$
f_{2}(x, t)=\frac{2 \sqrt{\Theta_{13}} \sinh \left(b_{6} t+b_{5} x\right)}{\sqrt{16 b_{5}^{6} b_{6}-b_{6}^{3}}}+c_{1} e^{\Theta_{14}}+c_{2} e^{-\Theta_{14}}
$$

Thus,

$$
\Xi_{2}(x, t)=\frac{6\left(\left(-\sqrt[3]{2} \sqrt[3]{b_{6}}-b_{5}\right) c_{1} e^{\Theta_{14}}+\left(\sqrt[3]{2} \sqrt[3]{b_{6}}+b_{5}\right) c_{2} e^{-\Theta_{14}}+\frac{2 b_{5} \sqrt{\Theta_{13}} \cosh \left(b_{6} t+b_{5} x\right)}{\sqrt{16 b_{5}^{6} b_{6}-b_{6}^{3}}}\right)}{\alpha\left(\frac{2 \sqrt{\Theta_{13}} \sinh \left(b_{6} t+b_{5} x\right)}{\sqrt{16 b_{5}^{6} b_{6}-b_{6}^{3}}}+c_{1} e^{\Theta_{14}}+c_{2} e^{-\Theta_{14}}\right)},
$$

where

$$
\begin{aligned}
\Theta_{13}= & -48 \sqrt[3]{2} b_{5}^{5} b_{6}^{4 / 3} c_{1} c_{2}-482^{2 / 3} b_{5}^{4} b_{6}^{5 / 3} c_{1} c_{2}+12 \sqrt[3]{2} b_{5}^{2} b_{6}^{7 / 3} c_{1} c_{2} \\
& +122^{2 / 3} b_{5} b_{6}^{8 / 3} c_{1} c_{2}+7 b_{6}^{3} c_{1} c_{2}-24 b_{5}^{3} b_{6}^{2} c_{1} c_{2}-16 b_{5}^{6} b_{6} c_{1} c_{2} \\
\Theta_{14}= & b_{6} t+\left(-\sqrt[3]{2} \sqrt[3]{b_{6}}-b_{5}\right) x
\end{aligned}
$$

\section{Numerical simulations}

In this section, using suitable values of parameters and different values of the coefficients of the nonlinear term in the studied equation, we present the dynamics of lump solution with the periodic and singular periodic wave solutions.

Figure 1 presents the interaction between lump, kink, and singular periodic (breather) wave solutions. Figure $1(\mathrm{a}, \mathrm{d})$ and (b,e) display lump-kink shape when $\alpha<0$ and Fig. 1 (c,f) displays lump-kink shape with some singularity when $\alpha>0$. Figure 2 presents the interaction between lump and kink (breather) solutions. Figure 2 (a,d), (b,e), and (c,f) display lump-kink solutions throughout the range of values $-10<\alpha<10$. Figure 3 presents the interaction between lump and periodic (rogue) wave solutions. Throughout the range of values $-10<\alpha<10$, Figs. 3 (a,d), (b,e), and (c,f) display the singular bell-type shape. Figure 4 presents the interaction between the lump and periodic (rogue) wave solutions. Throughout the range of values $-10<\alpha<10$, Figs. 4 (a,d), (b,e), and (c,f) display the lump-kink shape. Figure 5 presents the interaction between the lump and singular periodic (multiwave) wave solutions. Throughout the range of values $-10<\alpha<10$, Figs. 5 $(\mathrm{a}, \mathrm{d}),(\mathrm{b}, \mathrm{e})$, and $(\mathrm{c}, \mathrm{f})$ display the lump-period shape. Figure 6 presents the interaction between the lump, kink and periodic (multiwave) wave solutions. Throughout the range of values $-10<\alpha<10$, Figs. $6(\mathrm{a}, \mathrm{d}),(\mathrm{b}, \mathrm{e})$, and $(\mathrm{c}, \mathrm{f})$ display the lump-kink shape. 


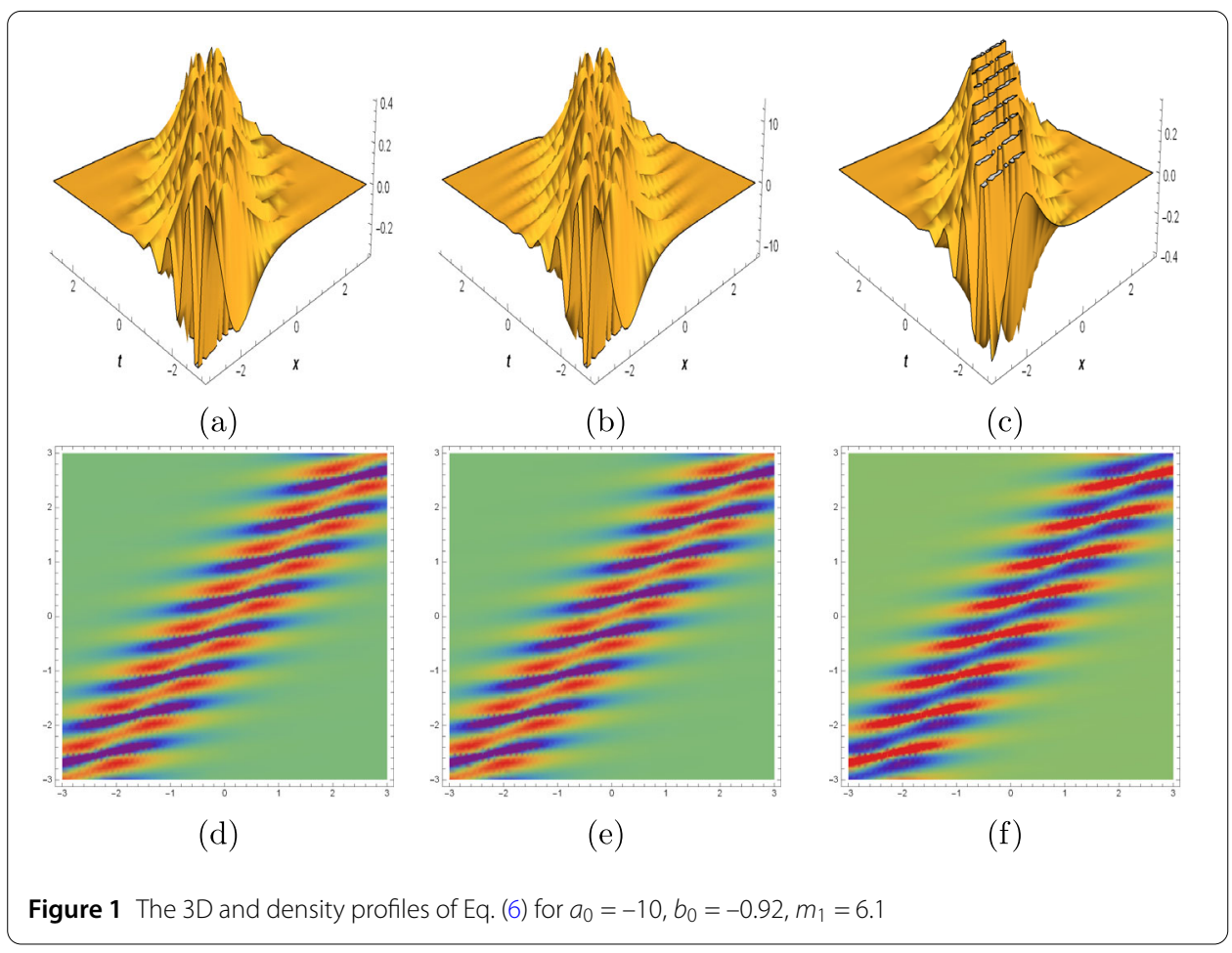

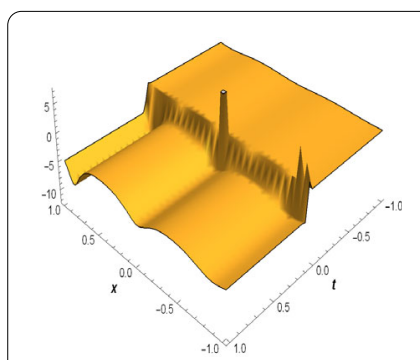

(a)

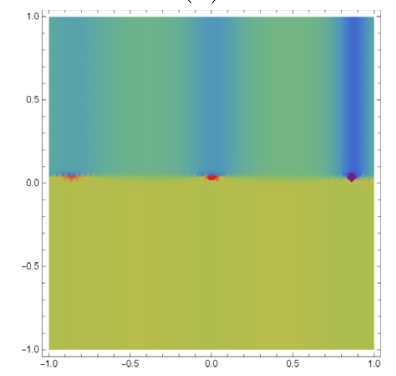

(d)

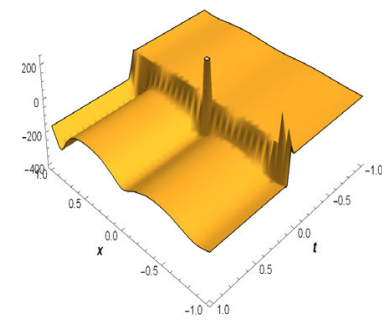

(b)

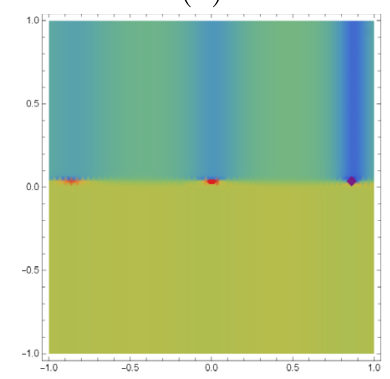

(e)

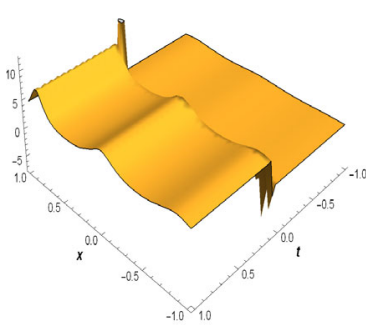

(c)

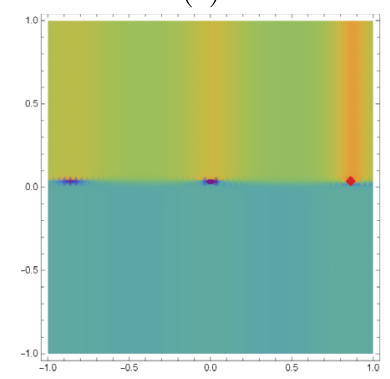

(f)

Figure 2 The 3D and density profiles of Eq. (8) for $p_{0}=-7.25, p_{1}=-0.26, m_{1}=-7.1$ 


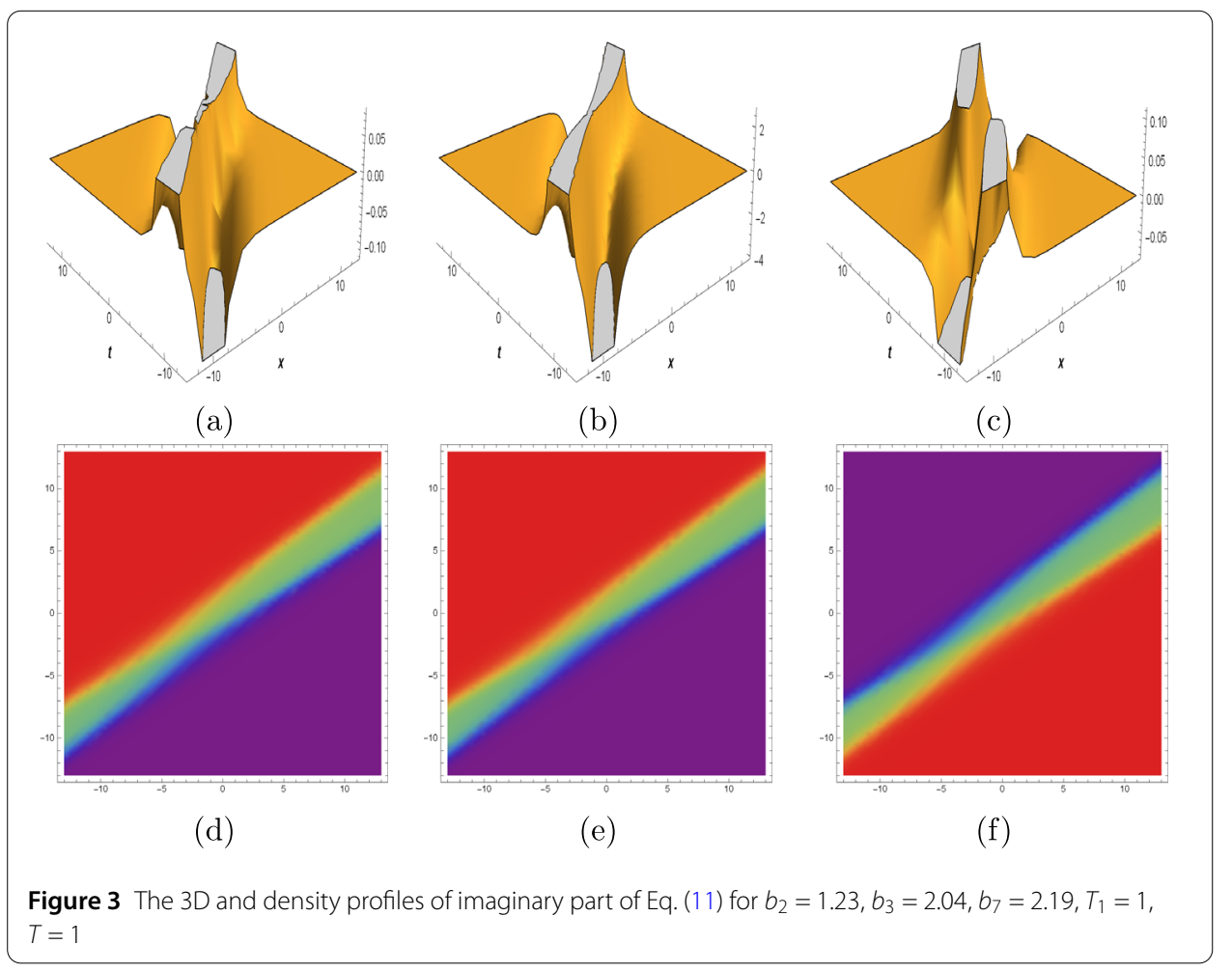

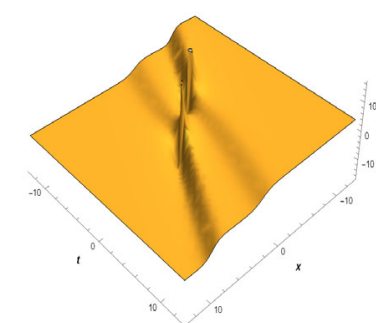

(a)

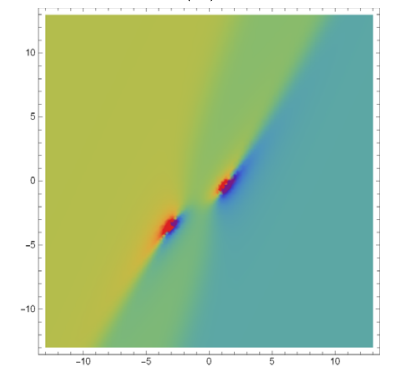

(d)

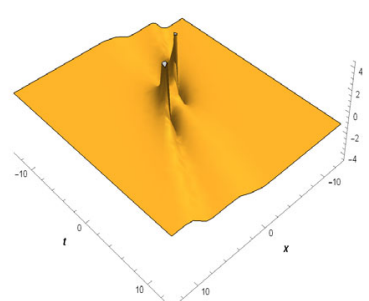

(b)

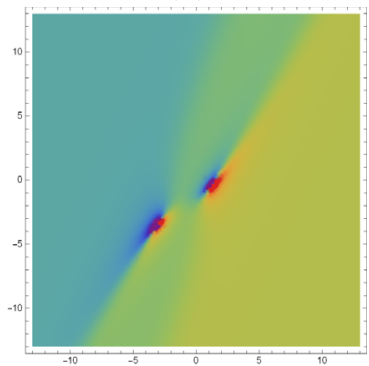

(e)

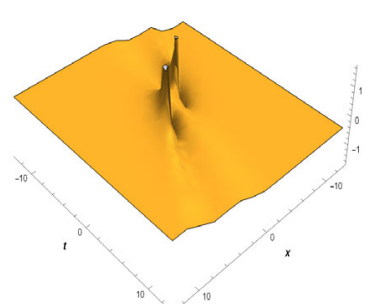

(c)

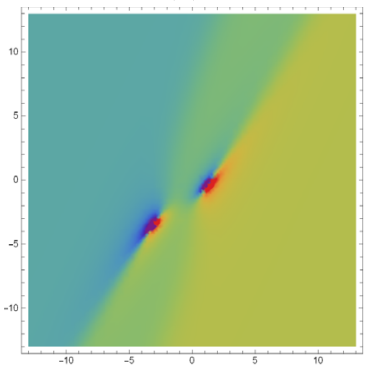

(f)

Figure 4 The 3D and density profiles of imaginary part of Eq. (11) for $b_{2}=1.23, b_{3}=2.04, b_{7}=2.19, T_{1}=1$, $T=1$ 


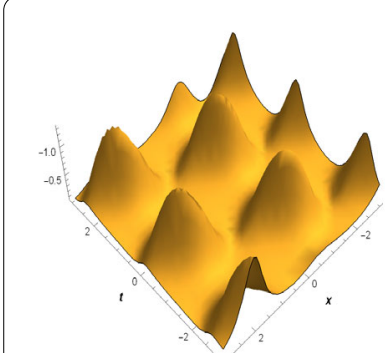

(a)

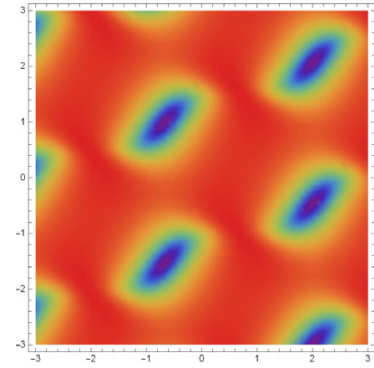

(d)

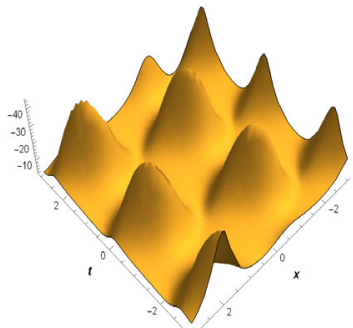

(b)

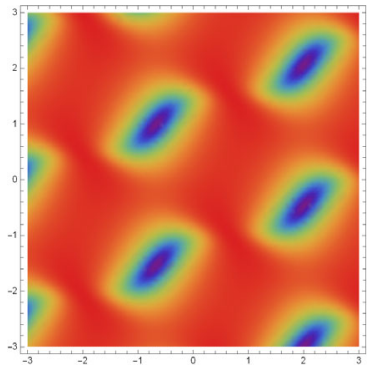

(e)

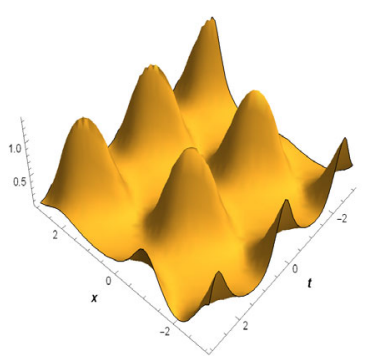

(c)

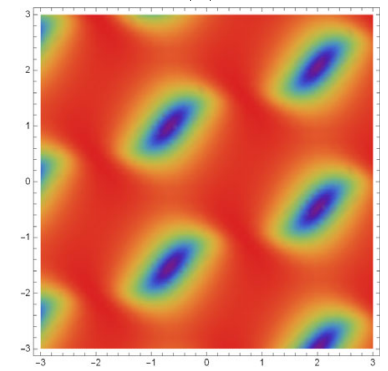

(f)

Figure 5 The 3D and density profiles of imaginary part of Eq. (14) for $c_{1}=1.04, b_{3}=2.62, c_{2}=2.38$

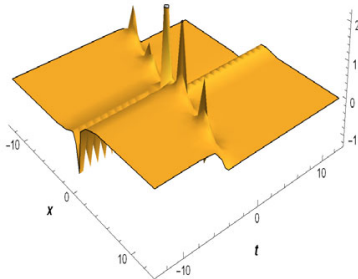

(a)

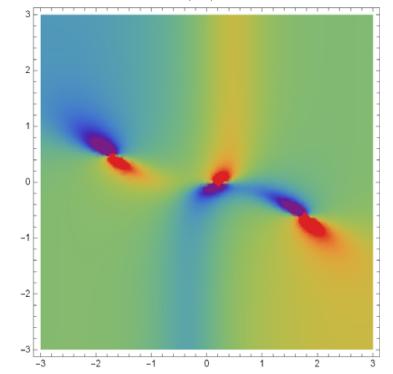

(d)

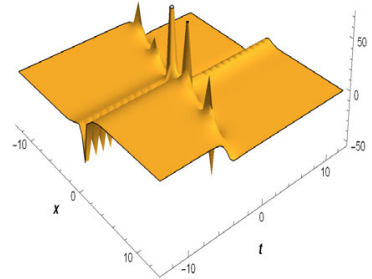

(b)

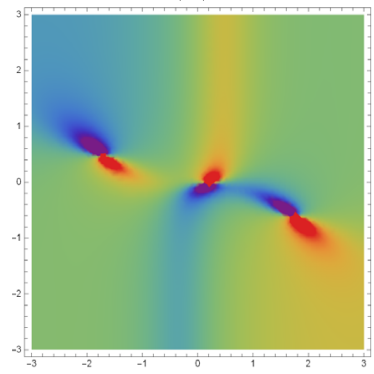

(e)

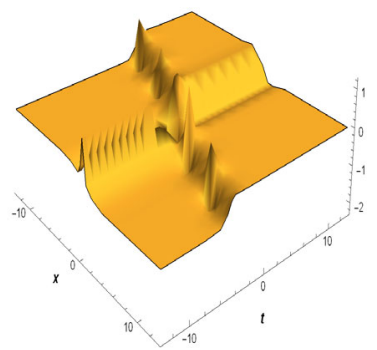

(c)

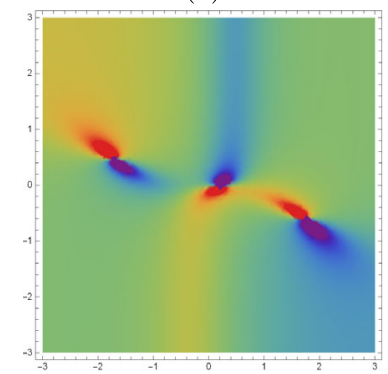

(f)

Figure 6 The 3D and density profiles of imaginary part of Eq. (16) for $c_{1}=0.74, b_{6}=-1, b_{5}=-1, c_{2}=-1$ 


\section{Invariant analysis}

The symmetries of (1) are expressed in the form of a vector field as

$$
\mathcal{X}=\xi_{1}(x, t, \Xi) \frac{\partial}{\partial x}+\xi_{2}(x, t, \Xi) \frac{\partial}{\partial t}+\xi_{3}(x, t, \Xi) \frac{\partial}{\partial \Xi}
$$

And the associated infinitesimals are:

$$
\begin{aligned}
& \xi_{1}=c_{1}-x c_{3}, \\
& \xi_{2}=c_{2}-3 t c_{3}, \\
& \xi_{3}=c_{4}+\Xi_{3},
\end{aligned}
$$

where $c_{i}(i=1,2,3,4)$ denote arbitrary constants. Consequently, (1) admits the following fields:

$$
\begin{aligned}
& \mathcal{X}_{1}=\partial_{x}, \\
& \mathcal{X}_{2}=\partial_{\Xi}, \\
& \mathcal{X}_{3}=\partial_{t}, \\
& \mathcal{X}_{4}=-3 t \partial_{t}+\Xi \partial_{\Xi}-x \partial_{x} .
\end{aligned}
$$

\subsection{Group of transformations}

The governing equation can be considered as a submanifold in the jet space $J^{3}\left(\mathcal{R}^{2}, \mathcal{R}^{2}\right)$. Therefore, to get the group transformations that the infinitesimal generators produce, $\eta_{1} \partial_{x}+\eta_{2} \partial_{t}+\eta_{3} \partial_{\Xi}$, the following systems of differential equations must be solved:

$$
\begin{array}{ll}
\frac{d \bar{x}(\epsilon)}{d \epsilon}=\xi_{1}(\bar{x}(\epsilon), \bar{t}(\epsilon), \bar{\Xi}(\epsilon)), & \bar{x}(0)=x, \\
\frac{d \bar{t}(\epsilon)}{d \epsilon}=\xi_{2}(\bar{x}(\epsilon), \bar{t}(\epsilon), \bar{\Xi}(\epsilon)), & \bar{t}(0)=t, \\
\frac{d \bar{\Xi}(\epsilon)}{d \epsilon}=\xi_{3}(\bar{x}(\epsilon), \bar{t}(\epsilon), \bar{\Xi}(\epsilon)), & \bar{\Xi}(0)=u .
\end{array}
$$

Taking the exponential of the obtained infinitesimal symmetries, the one-parameter groups $G_{k}(\epsilon)$ generated by $\mathcal{X}_{k}$ for $k=1, \ldots, 3$, are given by:

$$
\begin{aligned}
& \mathcal{G}_{1}:(x, t, \Xi) \rightarrow(x+\epsilon, t, \Xi), \\
& \mathcal{G}_{2}:(x, t, \Xi) \rightarrow(x, t+\epsilon, \Xi), \\
& \mathcal{G}_{3}:(x, t, \Xi) \rightarrow(x, t, \Xi+\epsilon), \\
& \mathcal{G}_{4}:(x, t, \Xi) \rightarrow\left(e^{-\epsilon} x, e^{-3 \epsilon} t, e^{\epsilon} \Xi\right),
\end{aligned}
$$

where entries give the transform point $e^{\epsilon \mathcal{X}_{i}}(x, t, \Xi)=(\bar{x}, \bar{t}, \bar{\Xi})$.

Note that in general a family of solutions, called invariant solutions, must refer to each parameter subgroup of the complete symmetry group of a system. We may therefore state the following: If $\Xi=f(x, t)$ is a solution for the governing equation, then such are the 
Table 1 The commutation relations of infinitesimal generators $\mathcal{X}_{1}, \mathcal{X}_{2}, \mathcal{X}_{3}$

\begin{tabular}{lllll}
\hline$\left[\mathcal{X}_{i}, \mathcal{X}_{j}\right]$ & $\mathcal{X}_{1}$ & $\mathcal{X}_{2}$ & $\mathcal{X}_{3}$ & $\mathcal{X}_{4}$ \\
\hline $\mathcal{X}_{1}$ & 0 & 0 & 0 & $-\mathcal{X}_{1}$ \\
$\mathcal{X}_{2}$ & 0 & 0 & 0 & $\mathcal{X}_{2}$ \\
$\mathcal{X}_{3}$ & 0 & 0 & 0 & $-3 \mathcal{X}_{3}$ \\
$\mathcal{X}_{4}$ & $\mathcal{X}_{1}$ & $-\mathcal{X}_{2}$ & $3 \mathcal{X}_{3}$ & 0 \\
\hline
\end{tabular}

following functions:

$$
\begin{aligned}
& \Xi_{1}=\mathcal{G}_{1}(\epsilon) \cdot f(x, t)=f(x+\epsilon, t), \\
& \Xi_{2}=\mathcal{G}_{2}(\epsilon) \cdot f(x, t)=f(x, t+\epsilon), \\
& \Xi_{3}=\mathcal{G}_{3}(\epsilon) \cdot f(x, t)=f\left(e^{-\epsilon} x, e^{-3 \epsilon} t\right),
\end{aligned}
$$

Now, one can get the general category of symmetries by considering a general linear combination $c_{1} \mathcal{X}_{1}+c_{2} \mathcal{X}_{2}+c_{3} \mathcal{X}_{3}+c_{4} \mathcal{X}_{4}$ of the given vector fields. In particular, if $\mathcal{G}$ is the symmetry group action near identity, it can be expressed in the form $\mathcal{G}=e^{\epsilon_{3} \mathcal{X}_{3}} \circ \cdots \circ e^{\epsilon_{1} \mathcal{X}_{1}}$.

\subsection{Commutator table}

Writing it in tabular form is the most convenient way of showing the structure of a given Lie algebra. Suppose that an $r$-dimensional Lie algebra is $g$ and $\mathcal{X}_{1}, \ldots, \mathcal{X}_{r}$ form a basis for $g$, then the commutator table for $g$ will be the $r \times r$ table whose $(i, j)$ th entry depicts the Lie bracket $\left[\mathcal{X}_{i}, \mathcal{X}_{j}\right]$. It should be noted that the table will remain skew-symmetric all the time since $\left[\mathcal{X}_{i}, \mathcal{X}_{j}\right]=-\left[\mathcal{X}_{j}, \mathcal{X}_{i}\right]$. Also, the structure constants can easily read off from the commutator table; namely, $C_{i j}^{k}$ is the coefficient of $\mathcal{X}_{k}$ in the $(i, j)$ th entry of Table 1.

\subsection{Adjoint representation tables}

Subsequently, the adjoint representation table is used to generate the adjoint transformations and to show the conjugacy map structure of the given Lie algebra. In the tabular form, it is useful to demonstrate conjugation relationships of each subalgebra with each other subalgebra. Define the adjoint operator as

$$
\operatorname{Ad}\left(e^{\epsilon \mathcal{X}}\right) \mathcal{Y} \equiv e^{-\epsilon \mathcal{X}} \mathcal{Y} e^{\epsilon \mathcal{X}}
$$

According to Campbell-Hausdorff [28], we have

$$
\operatorname{Ad}\left(e^{\epsilon \mathcal{X}}\right) \mathcal{Y}=\mathcal{Y}-\epsilon[\mathcal{X}, \mathcal{Y}]+\frac{\epsilon^{2}}{2}[\mathcal{Y},[\mathcal{X}, \mathcal{Y}]]-\cdots
$$

For an $n$-dimensional Lie algebra $\mathcal{L}^{n}$, the adjoint representation table is an $n \times n$ matrix, whose $(i, j)$ th entry presents the adjoint action of $\mathcal{X}_{i}$ on $\mathcal{X}_{j}$ as $\operatorname{Ad}\left(e^{\epsilon \mathcal{X}_{i}}\right) \mathcal{X}_{j}$. The adjoint representation table is given in Table 2.

\section{Adjoint system and conditions for nonlinear self-adjointness}

Take into account the following:

Theorem 5.1 Lie-Bäcklund, nonlocal, and Lie point symmetries, given by

$$
\mathcal{X}=\xi_{i} \frac{\partial}{\partial \bar{x}^{i}}+\eta_{\bar{\alpha}} \frac{\partial}{\partial \bar{\Xi}^{\bar{\alpha}}},
$$


Table 2 The commutation relations of infinitesimal generators $\mathcal{X}_{1}, \mathcal{X}_{2}, \mathcal{X}_{3}$

\begin{tabular}{lllll}
\hline$\left[\mathcal{X}_{i}, \mathcal{X}_{j}\right]$ & $\mathcal{X}_{1}$ & $\mathcal{X}_{2}$ & $\mathcal{X}_{3}$ & $\mathcal{X}_{4}$ \\
\hline $\mathcal{X}_{1}$ & $\mathcal{X}_{1}$ & $\mathcal{X}_{2}$ & $\mathcal{X}_{3}$ & $\epsilon \mathcal{X}_{1}+\mathcal{X}_{4}$ \\
$\mathcal{X}_{2}$ & $\mathcal{X}_{1}$ & $\mathcal{X}_{2}$ & $\mathcal{X}_{3}$ & $-\epsilon \mathcal{X}_{2}+\mathcal{X}_{4}$ \\
$\mathcal{X}_{3}$ & $\mathcal{X}_{1}$ & $\mathcal{X}_{2}$ & $\mathcal{X}_{3}$ & $3 \epsilon \mathcal{X}_{3}+\mathcal{X}_{4}$ \\
$\mathcal{X}_{4}$ & $e^{-\epsilon} \mathcal{X}_{1}$ & $e^{\epsilon} \mathcal{X}_{2}$ & $e^{-3 \epsilon} \mathcal{X}_{3}$ & $\mathcal{X}_{4}$ \\
\hline
\end{tabular}

of a nonlinear partial differential equation

$$
F_{\bar{\alpha}}\left(\bar{x}, \Xi, \ldots, \Xi_{s}\right)=0, \quad \bar{\alpha}=1,2, \ldots, \bar{m}
$$

with $m$ dependent variables will have an adjoint equation

$$
\mathcal{F}_{\bar{\alpha}}^{*}\left(\bar{x}, \Xi, \ldots, \Xi_{s}\right)=\frac{\delta\left(v^{\bar{\beta}} \mathcal{F}_{\bar{\beta}}\right)}{\delta \Xi^{\bar{\alpha}}}, \quad \bar{\alpha}=1,2, \ldots, \bar{m},
$$

the Lagrangian is thus

$$
\mathcal{L}=Z^{\bar{\beta}} \mathcal{F}_{\bar{\beta}}\left(\bar{x}, \Xi, \Xi_{(1)}, \ldots, \Xi_{(s)}\right),
$$

with $Z$ denotes a new dependent variable.

On account of (1), we have

$$
\mathcal{L}=v\left(\alpha\left(\Xi_{x} \Xi_{x t}+\Xi_{t} \Xi_{x x}\right)+\Xi_{t t}+\Xi_{x x x t}\right) .
$$

And the adjoint equation is then obtained as

$$
\mathcal{F}^{*}=\frac{\delta \mathcal{L}}{\delta \Xi}=0
$$

where

$$
\frac{\delta \mathcal{L}}{\delta \Xi}=\frac{\partial \mathcal{L}}{\partial \Xi}-D_{t} \frac{\partial \mathcal{L}}{\partial \Xi_{t}}-D_{x} \frac{\partial \mathcal{L}}{\partial \Xi_{x}}+\left(D_{x}\right)^{2} \frac{\partial \mathcal{L}}{\partial \Xi_{x x}}-\left(D_{x x x}\right)^{3} \frac{\partial \mathcal{L}}{\partial \Xi_{x x x}}+\left(D_{x}\right)^{4} \frac{\partial \mathcal{L}}{\partial \Xi_{x x x x}}
$$

On the basis of (29), we obtain

$$
\mathcal{F}^{*}=2 \alpha \Xi_{x t} v_{x}+\alpha \Xi_{x} v_{x t}+\alpha \Xi_{t} v_{x x}+v_{t t}+v_{x x x t}=0 .
$$

Definition 5.2 Equation (1) is an NSA only if

$$
\left.\mathcal{F}^{*}\right|_{v=Z(x, t, \Xi)}=\Lambda \mathcal{F}=0
$$

such that not all $v=Z(x, t, \Xi)$ are zero and $\Lambda_{i}(i=1,2,3)$ are undetermined coefficients.

Therefore, from the coefficients of $\Xi_{t}, \Xi_{x}, \Xi_{x t}, \Xi_{x x}, \Xi_{x x t}, \Xi_{x x x}$, we obtain

$$
\Lambda=-Z_{\Xi}
$$


Consequently, we reach the differential substitution as

$$
H=c_{1}+t c_{2} \text {. }
$$

Hence, (1) is an NSA.

\subsection{Conservation laws}

Herein, we establish the conservation laws of (1). We recall the following theorem:

Theorem 5.3 Equation(1) with obtained symmetries satisfies the conservation equation

$$
\left.D_{i}\left(C^{i}\right)\right|_{(1)=0}=0,
$$

where

$$
\begin{aligned}
C^{i}= & \xi_{i} \mathcal{L}+W^{\bar{\alpha}}\left[\frac{\partial \mathcal{L}}{\partial \Xi_{i}^{\bar{\alpha}}}-D_{j}\left(\frac{\partial \mathcal{L}}{\partial \Xi_{i j}^{\bar{\alpha}}}\right)+D_{j} D_{k}\left(\frac{\partial \mathcal{L}}{\partial \Xi_{i j k}^{\bar{\alpha}}}\right)-\cdots\right] \\
& +D_{j}\left(W^{\bar{\alpha}}\right)\left[\frac{\partial \mathcal{L}}{\partial \Xi_{i j}^{\bar{\alpha}}}-D_{k}\left(\frac{\partial \mathcal{L}}{\partial \Xi_{i j k}^{\bar{\alpha}}}\right)+\cdots\right]+D_{j} D_{k}\left(W^{\bar{\alpha}}\right)\left[\frac{\partial \mathcal{L}}{\partial \Xi_{i j k}^{\bar{\alpha}}}+\cdots\right]
\end{aligned}
$$

and $W^{\bar{\alpha}}=\eta_{\bar{\alpha}}-\xi_{j} \Xi_{j}^{\bar{\alpha}}$. The expression $C^{i}$ represents the conserved vectors.

Now, we compute the conservation laws for (1) using the obtained symmetries.

- The symmetry $\mathcal{X}_{1}=\partial_{x}$ admits the conserved vectors:

$$
\begin{aligned}
& C_{1}^{x}=\frac{1}{4}\left(\left(c_{1}+c_{2} t\right)\left(4 \alpha \Xi_{x} \Xi_{x t}+4 \Xi_{t t}+\Xi_{x x x t}\right)+c_{2}\left(2 \alpha \Xi_{x}^{2}+\Xi_{x x x}\right)\right), \\
& C_{1}^{t}=u_{x}\left(c_{2}-\alpha\left(c_{1}+c_{2} t\right) \Xi_{x x}\right)-\frac{1}{4}\left(c_{1}+c_{2} t\right)\left(4 \Xi_{x t}+\Xi_{x x x x}\right) .
\end{aligned}
$$

- The symmetry $\mathcal{X}_{2}=\partial_{\Xi}$ admits the conserved vectors

$$
\begin{aligned}
& C_{2}^{x}=-\frac{1}{2} \alpha\left(\left(c_{1}+c_{2} t\right) \Xi_{x t}+c_{2} \Xi_{x}\right), \\
& C_{2}^{t}=\frac{1}{2} \alpha\left(c_{1}+c_{2} t\right) \Xi_{x x}-c_{2} .
\end{aligned}
$$

- The symmetry $\mathcal{X}_{3}=\partial_{t}$ admits the conserved vectors

$$
\begin{aligned}
C_{3}^{x}= & \frac{1}{4}\left(\Xi_{t}\left(2 \alpha c_{2} \Xi_{x}-2 \alpha\left(c_{1}+c_{2} t\right) \Xi_{x t}\right)\right. \\
& \left.-\left(c_{1}+c_{2} t\right)\left(2 \alpha \Xi_{t t} \Xi_{x}+3 \Xi_{x x t t}\right)+c_{2} \Xi_{x x t}\right), \\
C_{3}^{t}= & \frac{1}{4}\left(2 \Xi_{t}\left(\alpha\left(c_{1}+c_{2} t\right) \Xi_{x x}+2 c_{2}\right)+\left(c_{1}+c_{2} t\right)\left(2 \alpha \Xi_{x} \Xi_{x t}+3 \Xi_{x x x t}\right)\right) .
\end{aligned}
$$


- The symmetry $\mathcal{X}_{4}=-3 t \partial_{t}+\Xi \partial_{\Xi}-x \partial_{x}$ admits the conserved vectors

$$
\begin{aligned}
C_{4}^{x}= & \frac{1}{4}\left(-2 \alpha\left(3 t \Xi_{t}+x \Xi_{x}+u\right)\left(\left(c_{1}+c_{2} t\right) \Xi_{x t}+c_{2} \Xi_{x}\right)\right. \\
& +2 \alpha\left(c_{1}+c_{2} t\right) \Xi_{1}\left(4 \Xi_{t}+3 t \Xi_{t t}+x \Xi_{x t}\right) \\
& +4 \alpha\left(c_{1}+c_{2} t\right) \Xi_{t}\left(2 \Xi_{x}+3 t \Xi_{x t}+x \Xi_{x x}\right) \\
& -4 x\left(c_{1}+c_{2} t\right)\left(\alpha \Xi_{x} \Xi_{x t}+\alpha \Xi_{t} \Xi_{x x}+\Xi_{t t}+\Xi_{x x x t}\right) \\
& \left.+3\left(c_{1}+c_{2} t\right)\left(6 \Xi_{x x t}+3 t \Xi_{x x t t}+x \Xi_{x x x t}\right)-c_{2}\left(3 \Xi_{x x}+3 t \Xi_{x x t}+x \Xi_{x x x}\right)\right), \\
C_{4}^{t}= & \frac{1}{4}\left(2 \alpha\left(c_{1}+c_{2} t\right) \Xi_{x}\left(2 \Xi_{x}+3 t \Xi_{x t}+x \Xi_{x x}\right)\right. \\
& -\left(3 t \Xi_{t}+x \Xi_{x}+\Xi\right)\left(4 c_{2}-2 \alpha\left(c_{1}+c_{2} t\right) \Xi_{x x}\right) \\
& -12 t\left(c_{1}+c_{2} t\right)\left(\alpha \Xi_{x} \Xi_{x t}+\alpha \Xi_{t} \Xi_{x x}+\Xi_{t t}+\Xi_{x x x t}\right) \\
& +4\left(c_{1}+c_{2} t\right)\left(4 \Xi_{t}+3 t \Xi_{t t}+x \Xi_{x t}\right) \\
& \left.+\left(c_{1}+c_{2} t\right)\left(4 \Xi_{x x x}+3 t \Xi_{x x x t}+x \Xi_{x x x x}\right)\right) .
\end{aligned}
$$

\section{Concluding remarks}

In this research, we investigated a new fourth-order integrable nonlinear equation by means of the efficient Hirota bilinear and Lie symmetry approaches. Consequently, we established novel types of solutions, such as breather, rogue and three-wave solutions. Wazwaz [28] investigated this new fourth-order integrable nonlinear equation. Multiple soliton solutions were reported using direct substitution. By using the tanh-coth method, topological and singular soliton solutions were constructed. On the other hand, using the tanh-coth method, singular periodic solutions were successfully reported. Comparing our solutions with the results presented in [28], one can observe that our results are new. To the best of our knowledge, the results reported in this paper have not been published, yet. Moreover, the reported solutions in this study have some physical meanings, for instance, the hyperbolic sine arises in the gravitational potential of a cylinder and the calculation of the Roche limit. The hyperbolic cosine function is the shape of a hanging cable (the socalled catenary) [36]. On the other hand, invariance properties, such as the group of transformations, as well as commutator and adjoint representation tables, have been reported. A differential substitution has been found via nonlinear self-adjointness and the associated conservation laws have been established. Using suitable values of parameters, the dynamical characteristics of the obtained solutions have been depicted via the 3-dimensional and contour graphs. To the best of our knowledge, the results and analysis presented in this study have not appeared in the literature before.

Acknowledgements

The Deanship of Scientific Research (DSR) at King Abdulaziz University, Jeddah, Saudi Arabia funded this project, under grant no. FP-28-42.

Funding

This research is supported by the King Abdulaziz University (FP-28-42).

Availability of data and materials

Not applicable.

Ethics approval and consent to participate

This article does not contain any studies with human participants or animals performed by any of the authors. 


\section{Competing interests}

The authors declare that they have no competing interests.

\section{Authors' contributions}

All of the authors contributed equally in writing this paper. They all read and approved the final manuscript.

\section{Author details}

${ }^{1}$ Department of Mathematics, Cankaya University, Ankara, 06530, Turkey. ${ }^{2}$ Institute of Space Sciences, Bucharest, Romania. ${ }^{3}$ Mathematical Modelling and Applied Computation Research Group (MMAC), Department of Mathematics, Faculty of Science, King Abdulaziz University, Jeddah 21589, Saudi Arabia.

\section{Publisher's Note}

Springer Nature remains neutral with regard to jurisdictional claims in published maps and institutional affiliations.

\section{Received: 28 September 2020 Accepted: 23 March 2021 Published online: 07 April 2021}

\section{References}

1. Kofane, T.C., Fokou, M., Mohamadou, A., Yomba, E.: Lump solutions and interaction phenomenon to the third-order nonlinear evolution equation. Eur. Phys. J. Plus 132(11), 465-473 (2017)

2. Zhang, L., Ma, W.X., Huang, Y.: Lump solutions of a nonlinear PDE combining with a new fourth-order term. Adv. Math. Phys. 2020, 3542320 (2020)

3. Satsuma, J., Ablowitz, M.J.: Two-dimensional lumps in nonlinear dispersive systems. J. Math. Phys. 20(7), 1496-1503 (1979)

4. Imai, K.: Dromion and lump solutions of the Ishimori-l equation. Prog. Theor. Phys. 98(5), 1013-1023 (1997)

5. Yang, J.Y., Ma, W.X.: Lump solutions to the BKP equation by symbolic computation. Int. J. Mod. Phys. B 30(28-29), $1640028(2016)$

6. Gilson, C.R., Nimmo, J.J.C.: Lump solutions of the BKP equation. Phys. Lett. A 147(8-9), 472-476 (1990)

7. Kaup, D.J.: The lump solutions and the Bäcklund transformation for the three-dimensional three-wave resonant interaction. J. Math. Phys. 22(6), 1176-1181 (1981)

8. Yong, X., Ma, W.X., Huang, Y., Liu, Y.: Lump solutions to the Kadomtsev-Petviashvili I equation with a self-consistent source. Comput. Math. Appl. 75(9), 3414-3419 (2018)

9. Ma, W.X., Zhou, Y., Dougherty, R.: Lump-type solutions to nonlinear differential equations derived from generalized bilinear equations. Int. J. Mod. Phys. B 30(28-29), 1640018 (2016)

10. Zhang, H.Q., Ma, W.X.: Lump solutions to the (2+1)-dimensional Sawada-Kotera equation. Nonlinear Dyn. 87(4), 2305-2310 (2017)

11. Ma, W.X.: A search for lump solutions to a combined fourth-order nonlinear PDE in (2+1)-dimensions. J. Appl. Anal. Comput. 9, 1319-1332 (2019)

12. Chen, S.T., Ma, W.X.: Lump solutions to a generalized Bogoyavlensky-Konopelchenko equation. Front. Math. China 13(3), 525-534 (2018)

13. Wang, C., Dai, Z., Liu, C.: Interaction between kink solitary wave and rogue wave for $(2+1)$-dimensional Burgers equation. Mediterr. J. Math. 13, 1087-1098 (2016)

14. Wazwaz, A.M.: New (3 + 1)-dimensional nonlinear evolution equation: multiple soliton solutions. Cent. Eur. J. Eng. 4, 352-356 (2014)

15. Lü, X., Ma, W.X., Chen, S.T., Khalique, C.M.: A note on rational solutions to a Hirota-Satsuma-like equation. Appl. Math. Lett. 58, 13-18 (2016)

16. Tang, Y., Tao, S., Guan, Q.: Lump solitons and the interaction phenomena of them for two classes of nonlinear evolution equations. Comput. Math. Appl. 72(9), 2334-2342 (2016)

17. Yang, J.Y., Ma, W.X.: Abundant lump-type solutions of the Jimbo-Miwa equation in (3 + 1)-dimensions. Comput. Math. Appl. 73(2), 220-225 (2017)

18. Yang, J.Y., Ma, W.X., Qin, Z.: Lump and lump-soliton solutions to the $(2+1)$-dimensional Ito equation. Anal. Math. Phys. 8(3), 427-436 (2018)

19. Ma, W.X.: Lump-type solutions to the (3 + 1)-dimensional Jimbo-Miwa equation. Int. J. Nonlinear Sci. Numer. Simul. 17(7-8), 355-359 (2017)

20. Anco, S.C., Bluman, G.: Direct construction of conservation laws from field equations. Phys. Rev. Lett. 78, 2869-2873 (1997)

21. Anco, S., Bluman, G.: Direct construction method for conservation laws of partial differential equations part l: examples of conservation law classifications. Eur. J. Appl. Math. 13, 545-566 (2002)

22. Anco, S., Bluman, G.: Direct construction method for conservation laws of partial differential equations part II: general treatment. Eur. J. Appl. Math. 13, 567-585 (2002)

23. Kara, A.H., Mahomed, F.M.: Noether-type symmetries and conservation laws via partial Lagrangians. Nonlinear Dyn. 45, 367-383 (2006)

24. Ibragimov, N.H.: A new conservation theorem. J. Math. Anal. Appl. 333, 311-328 (2007)

25. Miller, W: Symmetry Groups and Their Applications, vol. 50. Academic Press, San Diego (1972)

26. Dimas, S., Tsoubelis, D.: SYM: a new symmetry finding package for Mathematica. In: The 10th International Conference in MOdern GRoup ANalysis, Nicosia, pp. 64-70 (2005)

27. Aliyu, A.I., Baleanua, D., Inc, M., Yusuf, A.: Lie symmetry analysis, exact solutions and conservation laws for the time fractional modified Zakharov-Kuznetsov equation. Nonlinear Anal., Model. Control 22(6), 861-876 (2017)

28. Wazwaz, A.M.: Two new integrable fourth-order nonlinear equations: multiple solitons and multiple complex soliton solutions. Nonlinear Dyn. 94, 2655-2663 (2018)

29. Sulaiman, T.A., Yusuf, A.: Dynamics of lump-periodic and breather waves solutions with variable coefficients in liquid with gas bubbles. Waves Random Complex Media (2021). https://doi.org/10.1080/17455030.2021.1897708 
30. Hirota, R.: The Direct Method in Soliton Theory. Cambridge University Press, Cambridge (2004)

31. Foroutan, M., Manafian, J., Ranjbaran, A.: Lump solution and its interaction to $(3+1)$-D potential-YTSF equation. Nonlinear Dyn. 92, 2077-2092 (2018)

32. Sulaiman, T.A., Yusuf, A., Atangana, A.: New lump, lump-kink, breather waves and other interaction solutions to the $(3+1)$-dimensional soliton equation. Commun. Theor. Phys. 72, 085004 (2020)

33. Yusuf, A., Sulaiman, T.A., Inc, M., Bayram, M.: Breather wave, lump-periodic solutions and some other interaction phenomena to the Caudrey-Dodd-Gibbon equation. Eur. Phys. J. Plus 135, 563 (2020)

34. Wang, H.: Lump and interaction solutions to the $(2+1)$-dimensional Burgers equation. Appl. Math. Lett. 85, 27-34 (2018)

35. He, B., Meng, Q.: Bilinear form and new interaction solutions for the sixth-order Ramani equation. Appl. Math. Lett. 98 411-418 (2019)

36. Weisstein, E.W.: Concise Encyclopedia of Mathematics, 2nd edn. CRC Press, New York (2002)

\section{Submit your manuscript to a SpringerOpen ${ }^{\circ}$} journal and benefit from:

- Convenient online submission

- Rigorous peer review

- Open access: articles freely available online

- High visibility within the field

Retaining the copyright to your article

Submit your next manuscript at $\gg$ springeropen.com 